\title{
Clinical profile and prognostic indicators of tetanus in children
}

\author{
$\operatorname{Kosam} \mathbf{A}^{1}$, Durga $\mathbf{K}^{2}$, Kumar $\mathbf{H}^{3}$ \\ ${ }^{1}$ Dr Ajay Kosam, Associate Professor, Department of Pediatrics, ${ }^{2}$ Dr Durga Kosam, Assistant Professor, Department of \\ Anesthesiology and Critical Care, ${ }^{3}$ Dr Hemant Kumar, Professor and HOD, Dr Rakesh Nahrel, Associate Professor, \\ Department of Pediatrics, Chhattisgarh Institute of Medical Sciences, Bilaspur, CG, India.
}

Address for Correspondence: Dr Ajay Kosam, E mail: kosamajay@gmail.com

\begin{abstract}
Introduction: Tetanus is still a major health problem in developing countries. There is paucity of published data regarding pediatric tetanus in study area. This study was conducted to describe the clinical profile, management outcome and prognostic indicators of tetanus in children in our region. Material and Methods: This was a 5 year retrospective study of 24 pediatric tetanus patients < 18 years of age (excluding neonatal tetanus) from January 2010 to December 2014 admitted in Pediatric Intensive care unit of Chhattisgarh Institute of Medical Sciences Bilaspur (C.G.). Mean, summation, percentage and frequency distribution were used where applicable. Results: A total of 24 cases were evaluated. The male to female ratio was 1.4: 1 . The majority of patients $(79.1 \%)$ were $<10$ years of age. The mean age of cases were $6.94 \pm 3.02$ years (age range $21 / 2-12$ years). Only $25 \%$ children with tetanus had prior tetanus immunization. Post injury tetanus $(41.6 \%)$ and Otogenic tetanus $(37.5 \%)$ were the most common mode of infection. The average incubation period and period of onset were $7.5 \pm 3.13$ days and $3.2 \pm 0.96$ days respectively. Complication rate was $54.1 \%$. Mortality rate was $33.3 \%$. Incubation period $<5$ days, onset time $<3$ days, presence of autonomic dysfunction, need for mechanical ventilation and severity of tetanus were associated with higher mortality. Conclusion: Otogenic tetanus is a common mode of infection in children. Emphasis should be given to maintenance of ear hygiene and appropriate tetanus immunization in children with chronic ear discharge.
\end{abstract}

Keywords: Prognostic indicator of tetanus, Incubation Period, Tetanus, Time of Onset.

\section{Introduction}

Tetanus is a significant public health problem throughout the world. It is associated with a high morbidity and mortality particularly in the developing countries [1]. Tetanus cause approximately 213,000293,000 deaths worldwide each year, out of which 180,000 have been reported in neonates [2]. Tetanus is endemic in India. The annual incidence of neonatal tetanus in India is $1.74 / 1000$ live births [3]. The case fatality rate of tetanus ranges from $6 \%$ to $72 \%$ depending on the availability of well equipped intensive care unit [1].

In developed world tetanus occurs mainly in elderly because of decline in protective antibody levels [4]. In developing countries tetanus is common in children and young due to lack of effective immunization program and appropriate treatment of injuries [5].

Manuscript received: $4^{\text {th }}$ June 2015

Reviewed: $24^{\text {th }}$ June 2015

Author Corrected: $2^{\text {nd }}$ July 2015

Accepted for Publication: $11^{\text {th }}$ July 2015
Tetanus is caused by Clostridium Tetani. It is a gram positive, anaerobic, spore forming bacterium. It is found in soil and in animal and human faeces. The usual mode of entry is through a punctured wounds or lacerations. Tetanus may develop after CSOM, burns, gangrene, chronic ulcers, dog bites, dental infection, abortion and childbirth [1]. In some patients portal of entry for the organism cannot be identified [6].

Tetanus occurs sporadically. It affects unimmunized and partially immunized subjects. Completely immunized subjects who did not receive booster doses of vaccine may also be affected [7]. It is therefore very important that all age groups have the universal primary immunization with subsequent maintenance of adequate antitoxin levels by means of appropriately timed boosters [7].

The defined predictors of mortality in tetanus are short incubation period (less than 7 days), short time of onset (less than 3 days), autonomic dysfunction and cephalic 
tetanus [8]. There is a paucity of studies on tetanus from developing countries especially in pediatric patients. Therefore, this study was undertaken to analyze the clinical profile, predictors of mortality and outcome of pediatric tetanus cases in our region.

\section{Material and Methods}

\section{Study setting and design}

This was a 5 year retrospective study of 24 pediatric tetanus patients from January 2010 to December 2014 admitted in Pediatric Intensive care unit of Chhattisgarh Institute of Medical Sciences Bilaspur (C.G.)

\section{Study subjects}

24 pediatric tetanus patients $<18$ years of age excluding neonatal tetanus were analyzed. Clinical diagnosis of tetanus was based on the presence of one or more of the following:-

1. Trismus

2. Rigidity of the neck and or abdomen

3. Reflex spasms with a clear sensorium.

Tetanus was classified as generalized, cephalic, localized types. Localized tetanus was diagnosed if the patients had rigidity or spasm limited to the wound bearing area of the body. Children were classified as having generalized tetanus if there was trismus and generalized rigidity with or without generalized spasms. A form of localized tetanus restricted to head and neck was classified as cephalic tetanus.

Ablett classification [9] was used to grade the severity of tetanus as follows-

\begin{tabular}{|l|l|l|}
\hline Grade & Severity & Clinical features \\
\hline I & Mild & $\begin{array}{l}\text { Mild to moderate trismus; general spasticity; no respiratory embarrassment; no } \\
\text { spasms; little or no dysphagia }\end{array}$ \\
\hline II & Moderate & $\begin{array}{l}\text { Moderate trismus; well-marked rigidity; mild to moderate but short spasms; moderate } \\
\text { respiratory embarrassment with an increased respiratory rate }>30, \text { mild dysphagia }\end{array}$ \\
\hline III & Severe & $\begin{array}{l}\text { Severe trismus; generalized spasticity; reflex prolonged spasms; respiratory rate }>40 ; \\
\text { apnoeic spells, severe dysphagia; tachycardia }>120\end{array}$ \\
\hline
\end{tabular}

The treatment was started immediately once the diagnosis was made. The patients were treated as per standard protocol for the management of tetanus. The management included antibiotics (i.e. Penicillin or Metronidazole), wound care, human tetanus immune globulins (500 Units I/M stat) and active immunization with injection Tetanus Toxiod at the time of admission. Tetanus Toxoid dose was repeated when patient was discharged. Diazepam, Chlorpromazine and Magnesium sulphate was given for the control of spasm. Mechanical ventilation was instituted when required.

Following details were noted from medical records - demographic profile, history of tetanus immunization, mode of infection, incubation period, period of onset, clinical features, management, complications, duration of intensive care unit admission, outcome and prognostic factors.

Incubation period was defined as the time from exposure to the appearance of symptoms. The period of onset was defined as the interval between the first symptom and the first spasm. Descriptive statistics was used for data analysis.

\section{Results}

\section{Demographic data}

In our study 24 pediatric tetanus cases were evaluated. The mean age of cases were $6.94 \pm 3.02$ years (age range $21 / 2-12$ years). There were $58.3 \%$ boys and $41.7 \%$ girls. The mean age of males and females was $7.75 \pm 3.13$ and $5.78 \pm 2.63$ 
years respectively. $37.5 \%$ (9/24) children with tetanus were $\leq 5$ years of age, $41.7 \%(10 / 24)$ children with tetanus were in the age group of $6-10$ years and $20.8 \%(5 / 20)$ children with tetanus were $>10$ years of age (Table 1$)$

Table 1: Demographic profile of cases $(n=24)$

\begin{tabular}{|l|l|l|}
\hline S. No. & Variable & Value \\
\hline \multirow{4}{*}{2} & Age $(\mathbf{y r})$ & \\
\cline { 2 - 3 } & Mean, SD & $6.94 \pm 3.02$ \\
\hline \multirow{5}{*}{3} & Sex & 14 \\
\cline { 2 - 3 } & Male & 10 \\
\cline { 2 - 3 } & Female & \\
\hline & Age group & 9 \\
\cline { 2 - 3 } & $\leq 5$ years & 10 \\
\cline { 2 - 3 } & $6-10$ years & 5 \\
\cline { 2 - 3 } & $>10$ years & 10 \\
\hline
\end{tabular}

\section{Tetanus immunization status}

$18 / 24(75 \%)$ children with tetanus were not vaccinated or their tetanus immunization status was unknown. Only $6 / 24$ $(25 \%)$ children with tetanus had prior tetanus immunization. However, in patients who had prior tetanus immunization there was no documented proof of the immunization schedule in any cases. Thus all children with tetanus were either unimmunized or partially immunized.

\section{Portal of entry}

Trauma such us prick, puncture and laceration causing wound was the most common cause of tetanus in $41.66 \%$ of cases and commonly occurred in the lower limbs. Otogenic tetanus secondary to CSOM accounted for $37.5 \%$ cases of tetanus in children. The portals of entry was not identified in $20.8 \%$ of cases (Pie chart 1 )

\section{Pie chart 1: Portal of entry}

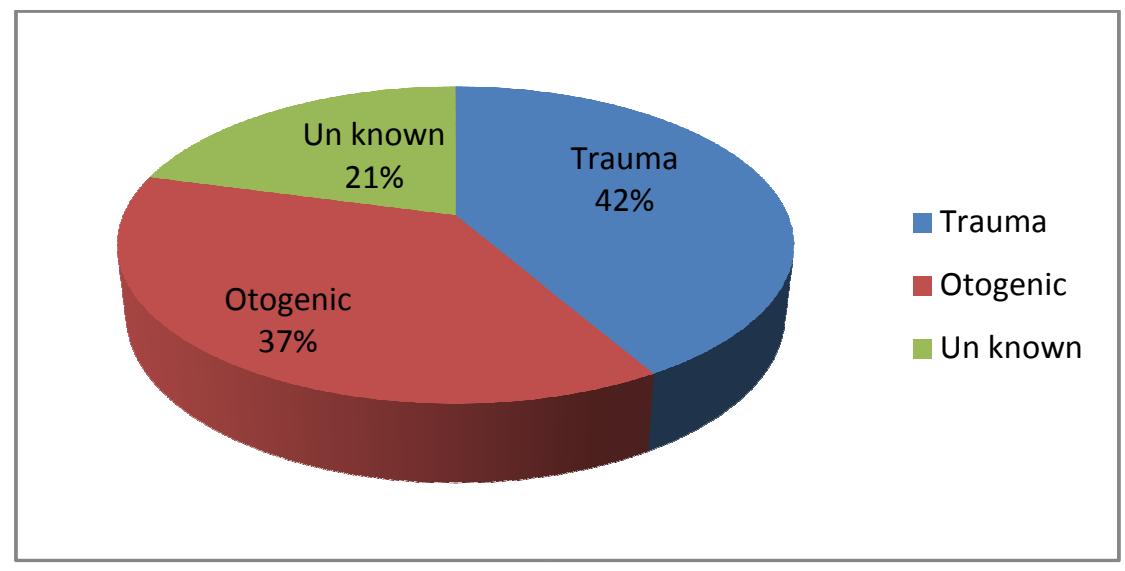

\section{Incubation period and period of onset}

The incubation period was known in only 10/24 (41.66\%) patients whereas in 14/24 (58.3\%) children with tetanus the incubation period was unknown. The mean incubation period was $7.5 \pm 3.13$ days. The majority of patients, $17 / 24$ (70.8\%) had incubation period of less than 7 days and 16 of them had severe disease. The period of onset (interval between the first symptom and the first spasm), was documented in 14/24 (58\%) cases. The mean onset period was $3.2 \pm$ 0.96 days (range 2-5 days).

\section{Clinical presentation}


All patients had generalized tetanus. No cases of cephalic and localized tetanus were recorded. According to Ablett classification for assessment of severity of tetanus $15(62.5 \%)$ children had severe disease, $8(33.3 \%)$ children had moderate disease and $1(4.1 \%)$ patient had very severe disease. The most common presenting complaints were body stiffness/spasm (100\%), trismus (100\%) and dysphagia (54.1\%) (Table 2).

Table 2: Clinical presentation of tetanus $(n=24)$

\begin{tabular}{|l|l|l|}
\hline Clinical presentation & Number of patients & Percentage \\
\hline Body stiffness/spasm & 24 & 100 \\
\hline Trismus & 24 & 100 \\
\hline Dysphagia & 13 & 54.1 \\
\hline Abdominal pain & 3 & 12.5 \\
\hline Body ache & 2 & 8.3 \\
\hline Fever & 2 & 8.3 \\
\hline Dyspnea & 2 & 8.3 \\
\hline
\end{tabular}

\section{Complications}

Complications of tetanus were documented in 13(54.1\%) children. Respiratory complications (pneumonia, respiratory failure) in $8(33.3 \%)$, cardiovascular (tachycardia, hypertension) in $4(16.6 \%)$ and gastrointestinal complications (paralytic ileus, GI bleed) in $3(12.5 \%)$ were documented in children with tetanus.

\section{Outcome of tetanus cases}

$8(33.3 \%)$ deaths were reported. Remaining $16(66.7 \%)$ children were discharged without any neurological disability. Majority of the deaths occurred within the first few days of hospitalization. 7 (87.5\%) children died in the first 7 days while $1(12.5 \%)$ child died after $7^{\text {th }}$ day. The mean duration of hospitalization for non-survivors was $4.75 \pm 3.52$ days (range 1-12 days).

\section{Mortality predictors}

Incubation period of tetanus in children in mortality group and survival group was $4.5 \pm 1.28$ days and $9.5 \pm 2.16$ days respectively. The time of onset was $2.66 \pm 0.50$ days in mortality group as compared to $4 \pm 0.75$ days in survival group. Mean age of children in mortality group was $8.3 \pm 2.65$ days whereas in survival group it was $6.71 \pm 2.3$ days. Autonomic dysfunction was seen in $37.5 \%$ children in mortality group whereas $12.5 \%$ children in survival group developed autonomic dysfunction. All children with moderate tetanus survived whereas $46.7 \%$ children with severe tetanus and $1(100 \%)$ child with very severe tetanus died. All children who required ventilatory support died. Short incubation period, short time of onset, presence of autonomic dysfunction, need for ventilatory support and increasing severity of tetanus were predictors associated with mortality in children with tetanus (Table 3 )

Table 3: Mortality predictors

\begin{tabular}{|l|l|l|}
\hline Predictor variable & Survival group $(\mathbf{n = 1 6})$ & Mortality group $(\mathbf{n}=\mathbf{8})$ \\
\hline Incubation period (days) & $9.5 \pm 2.16$ & $4.5 \pm 1.28$ \\
\hline Onset time (days ) & $4 \pm 0.75$ & $2.66 \pm 0.50$ \\
\hline Age & $6.71 \pm 2.83$ & $8.3 \pm 2.65$ \\
\hline Sex - Male & $9(56.25 \%)$ & $5(62.5 \%)$ \\
\hline Autonomic dysfunction & $2(12.5 \%)$ & $3(37.5 \%)$ \\
\hline Mechanical Ventilation & 0 & $4(50 \%)$ \\
\hline Severity of Tetanus & & \\
\hline Moderate & $8(100 \%)$ & 0 \\
\hline Severe & $8(55.3 \%)$ & $7(46.7 \%)$ \\
\hline Very severe & 0 & $1(100 \%)$ \\
\hline
\end{tabular}




\section{Discussion}

Tetanus is still prevalent in developing countries. It constitutes significantly to high morbidity and mortality despite the documented effectiveness and easy availability of tetanus vaccines [1]. High incidence of pediatric tetanus cases in developing countries is attributed to low primary immunization coverage and lack of adequate booster doses [5]. This observation is reflected in our study as three quarters of our patients were not vaccinated or their tetanus immunization status was unknown. This observation highlights the urgent need to strengthen preventive measures to reduce the incidence of tetanus. These measures include wide immunization coverage and health education.

Male preponderance and higher incidence of tetanus in children $<10$ years was observed in our study. Similar results have been documented in other studies $[4,6,10]$. The male preponderance could be explained by the fact that boys tend to spend more time outdoor hence they are more likely to be exposed to the causal organism. They are also more prone to penetrating injury thereby facilitating the organism to enter the body. Higher incidence of tetanus in younger children can be explained by the lack of effective immunization program and inappropriate treatment of injuries [5] whereas in developed countries tetanus occurs mainly in elderly due to decline in protective antibodies [4].

The most common mode of infection was post injury tetanus (42\%) followed by Otogenic tetanus (37\%). Mode of infection could not be ascertained in $21 \%$ cases. The incidence of Otogenic tetanus was high in our study as chronic otitis media and practice of local instillation of oil in the ear is common. Other factor that could contribute to Otogenic tetanus is introduction of unclean fingers and contaminated objects in the ear. Incidence of Otogenic tetanus in various studies range from $10.8 \%$ to $55 \%[11,12]$. Post injury tetanus is more common due to increased outdoor activities in children and neglect of injury. The mode of infection was not identified in significant number of cases reflecting that the injuries were likely to be trivial to be recalled. Trivial wounds as possible portal of entry for tetanus infection are common because most children in the rural areas do not wear shoes.

Body stiffness/spasm, trismus and dysphagia were the commonest complaints of the tetanus patients in our study. This finding is in agreement with other studies $[13,14]$. Hence, there should be a high index of suspicion for tetanus whenever patients present with any of these symptoms as tetanus is essentially a clinical diagnosis and laboratory tests as well as cultures have little diagnostic importance [15].

Complications like pneumonia, autonomic instability, GI bleed, hypoxemia, sepsis, urinary tract infections, decubitus ulcer and vertebral fractures have been reported in several studies [12, 16-18].Complications are contributory factor for high tetanus mortality $[16,18]$. Autonomic instability in tetanus is due to the effect of tetanus toxin on the brainstem and autonomic interneurones thereby impairing baroreceptor reflexes causing refractory hypotension. Direct effect of toxin on myocardium and loss of adrenal inhibition is another postulated mechanism for autonomic instability $[19,20]$. LRTI is common in tetanus patients due to suppression of protective airway reflexes and pooling of secretions secondary to heavy sedation and mechanical ventilation. Complication rate in our study was high $(54.1 \%)$. The most common complication in our study was respiratory infection followed by cardiovascular and gastrointestinal complications. Similar results have been documented in other studies $[4,6]$.

Prognosis of patients with tetanus varies depending on the age, grade of tetanus, availability of intensive care, complications etc. Overall, mortality is approximately $10-39.3 \%$ in various studies $[11,12,16,18]$. In our study, mortality rate was $33.3 \%$. Most of the deaths in our study were attributed to pneumonia, sudden cardiac arrest and respiratory failure, an observation similar to study by Feroz et al [6].The high mortality rate in this study is due to late referral and presence of complications at the time of admission to our unit. The poor prognostic factors in this study included shorter incubation periods $(<5$ days), short onset time $(<3$ days), presence of autonomic dysfunction, need for mechanical ventilation and severity of tetanus. Similar observations have been reported in other studies [21, 22]. In our study mechanical ventilation appeared to be a risk factor for mortality. This could be a confounding factor indicating severity as only the sicker patients were considered for mechanical ventilation.

\section{Conclusion}

Tetanus is a vaccine preventable infectious disease. Its prevalence is still high in our region with a high morbidity and mortality rate, even with advanced 
intensive care facilities for its management. Otogenic tetanus is a common mode of infection in children. Emphasis should be given to maintenance of ear hygiene and appropriate tetanus immunization in children with chronic ear discharge.

\section{Funding: Nil \\ Conflict of interest: Nil \\ Permission from IRB: Yes}

\section{References}

1. Oladiran I, Meier DE, Ojelade AA, Olaolorun DA, Adeniran A, Tarpley JL. Tetanus: continuing problem in the developing world. World J Surg 2002 Oct; 26(10):1282-85.

2. Vandelaer J, Birmingham M, Gasse F, Kurian M, Shaw C, Garnier S. Tetanus in developing countries: an update on the Maternal and Neonatal Tetanus Elimination Initiative. Vaccine 2003 Jul; 21(24):34425.

3. Singh J, Datta KK, Foster SO. Sensitivity of neonatal tetanus surveillance system in India. Indian Pediatr1997 May; 34(5):398-401.

4. Amare A, Yami A. Case-fatality of adult tetanus at Jimma University Teaching Hospital, Southwest Ethiopia. Afr Health Sci 2011 March; 11(1):36-40.

5. Dietz V, Milstien JB, Van Loon F, Cochi S, Bennett J. Performance and potency of tetanus toxoid: implications for eliminating neonatal tetanus. Bull World Health Organ 1996; 74(6):619-28.

6. Feroz AHM, Rahman MH. A Ten-year Retrospective Study of Tetanus at a Teaching hospital in Bangladesh. J Bangladesh Coll Phys Surg 2007 May; 25 (2):62-69.

7. Edlich RF, Hill LG, Mahler CA, Cox MJ, Becker DG, Horowitz JH. Management and prevention of tetanus. J Long Term Eff Med Implants 2003; 13(3):139-54.

8. Arnon SS. Tetanus (Clostridium tetani). In: Behrman RE, Kleigman RM, Jenson HB, Stanton BF, editors. Nelson Textbook of Pediatrics. 18th ed. Philadelphia: Saunders; 2007:1228-30
9. Ablett JJL. Analysis and main experience in 82 patients treated in the Leeds tetanus unit. In: Ellis M, ed. Symposium on tetanus in Great Britain. Boston Spa UK: National Lending Library; 1967:1-10.

10. Joshi S, Agarwal B, Malla G, Karmacharya B. Complete elimination of tetanus is still elusive in developing countries: a review of adult tetanus cases from referral hospital in Eastern Nepal. Kathmandu Univ Med J 2007 Jul-Sep; 5(3):378-81.

11. Oyelami OA, Aladekomo TA, Ononye FO. A 10 year retrospective evaluation of cases of post-neonatal tetanus seen in a pediatric unit of a university teaching hospital in southwestern Nigeria (1985 to 1994). Cent Afr J Med 1996 Mar; 42(3): 73-5.

12. Mondal T, Aneja S, Tyagi A, Kumar P, Sharma D. A study of childhood tetanus in post-neonatal age group in Delhi. Indian Pediatr 1994 Nov; 31(11): 1369-72.

13. Lau LG, Kong KO, Chew PH: A ten-year retrospective study of tetanus at a general hospital in Malaysia. Singapore Med J 2001Aug; 42(8):346-50.

14. Younas NJ, Abro AH, Das K, Abdou AMS, Ustadi AM, Afzal S: Tetanus: Presentation and outcome in adults. Pak J Med Sci 2009 Oct-Dec; 25(5):760-765.

15. Sandford JP: Tetanus-Forgotten but not gone. N Engl J Med 1995 Mar; 332(12):812-3.

16. Wesley AG, Pather M. Tetanus in children: An 11 year review. Ann Trop Paediatr 1987 Mar; 7(1):32-37.

17. Peetermans WE, Schepens D. Tetanus - still a topic of present interest: A report of 27 cases from a Belgian referral hospital. J Intern Med 1996 Mar; 239 (3):24952.

18. Harding - Goldson HE, Hanna WJ. Tetanus: A recurring intensive care problem. J Trop Med Hyg 1995 Jun; 98 (3): 179-84.

19. Sun KO, Chan YW, Cheung RT, So PC, Yu YL, Li PC. Management of tetanus: A review of 18 cases. J R Soc Med 1994 Mar; 87(3): 135-7.

20. Bleck TP. Management of tetanus: A review of 18 cases. R Soc Med 1994 Sep; 87(9): 569. 
21. Bhatia R, Prabhakar S, Grover VK. Tetanus. Neurol India 2002 Dec;50(4):398-407
22. Cook TM, Protheoze RT, Handel JM. Tetanus: A review of literature. Br J Anaesth. 2001Sep; 87(3):47787.

\section{How to cite this article?}

Kosam A, Durga K, Kumar H. Clinical profile and prognostic indicators of tetanus in children. Int J Med Res Rev 2015;3(6):601-607. doi: 10.17511/ijmrr.2015.i6.117. 